\title{
Design of Online Training System for Power Enterprise
}

\author{
Lin Liu $^{\mathrm{a}, *}$, Xian Chen ${ }^{\mathrm{b}}$, Bo Chen, Xiaozi Cui \\ State Grid Henan Electric Power Company Luoyang Power Supply Company, Luoyang 471000, \\ China \\ aluoyangdidiao@sina.com, b1015903240@qq.com
}

Keywords: Electricity companies, online training, efficiency.

\begin{abstract}
With the rapid development of computer technology, the Internet has penetrated into people's work and life in social. Information is showing a strong vitality in production and operation of electricity enterprises. It has become the only way for the development of electric power enterprise. In the context of the smart grid, the training of traditional power companies also requires information and intelligence. The online training system for electricity enterprises not only can improve the efficiency of electricity enterprises, but also can save the costs of enterprise training.
\end{abstract}

\section{Introduction}

With the rapid development of the power system, the application of new technologies in production and large-capacity turbines to make electricity sector monitoring and operation technology continues to improve, increasing the operating personnel with extensive operating experience requirements. Due to the characteristics of the power system itself, staff and technical personnel working in the power industry, we must first undergo a rigorous professional knowledge, then through job training, become familiar with the work-site systems and equipment. Excellent technician can run a large extent avoids accidents, so the staff training has been the focus of domestic power industry.

Currently, modern computer networks, communications, database and multimedia-based information technology has been widely expanded in the field of industrial engineering, applied in wind farm has gradually entered a mature stage. MIS (Management Information System, MIS) has been widely used in the power industry in our country. MIS provides for electricity production, management, decision support, and office automation and other aspects of a comprehensive, accurate and timely information and a variety of data to speed up the communication of information between departments, thereby improving the efficiency of the power companies of various departments and reliable, so that all departments fast and efficient decision-making becomes possible to dramatically increase the economic benefits of electricity production and management level.

\section{The Technology Used in System Development}

The Online Training System for electricity enterprises is adopted B / S (browser, server) architecture development. The B / S structure is based on the browser, the client without having to buy any special software, use your browser to access online training system through the network; you can make training who break the constraints of time and place. Microsoft.Net Framework based systems to develop an ASP.NET, ASP.NET is a programming framework established in the common language runtime and can be used to build powerful Web applications on the server. In database management systems according to the demand characteristics of the system's functionality, using more extensive use of MS SQL Server 2008 database, use it with a cross-platform, scalable components, protocols, GM and other features; you can easily achieve online training system maintenance and secondary development.

Use B / S model development system that allows the separation procedures and operating systems, the development process need to consider the difference between different operating systems, 
reducing the complexity of development, but also more conducive to the management and expansion of the system.

The system uses Microsoft's .NET technology, combined with SQL Server 2008 relational database development. The system uses three-tier system: the presentation layer, application logic layer, data layer. Three-tier architecture logically independent of each other, in which a layer of modified time, the other layers will not have an impact. Because of the independence between the layers of the three-tier system can achieve respectively, corresponding respectively: client, application server, database server.

\section{System Goals}

According to the actual situation the plant network environment, using a centralized management model. Establish a data center in the Information Center of the plant room, employees use their own power plants or room access a central database via a PC network logon system for learning and training.

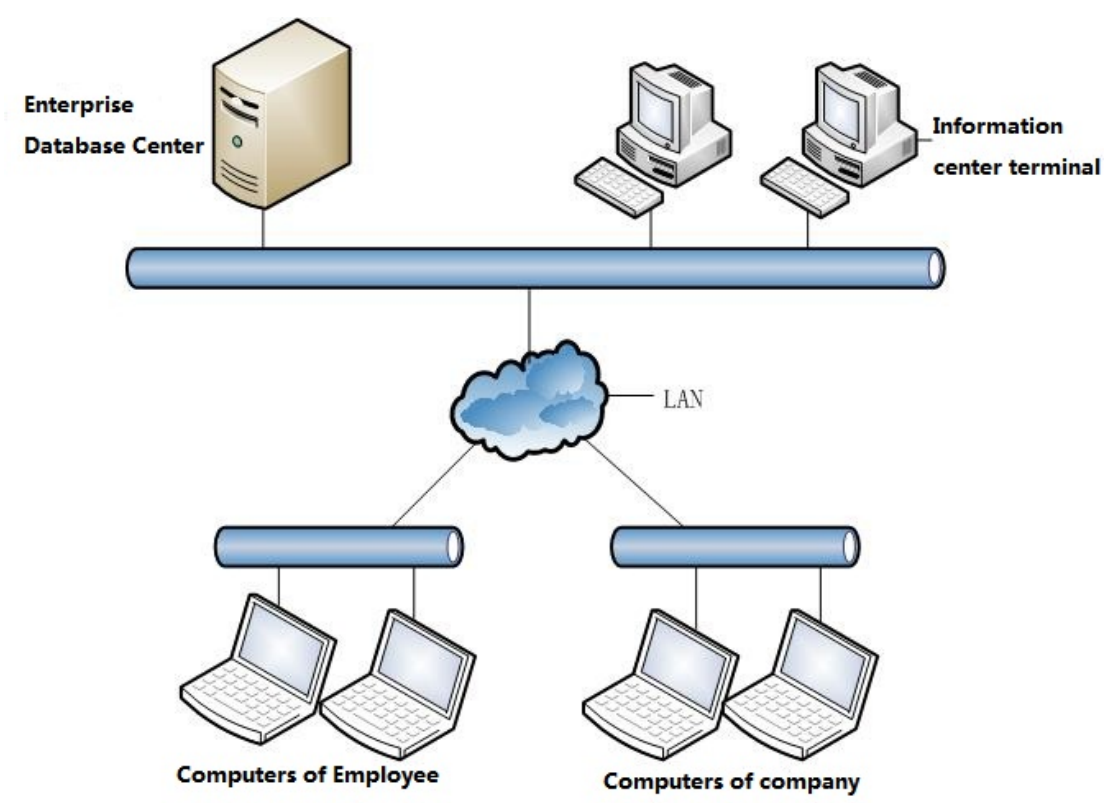

Figure1: The Structure of Power corporate online training system

Use the online training system for electricity enterprises, set the goal of building the following:

(1) According to the training needs of the actual production of the plant's operations to develop online training system, effectively improve the professional skills of employees of the plant, reduce training costs, improve training efficiency of the plant.

(2) Online training system for staff training and assessment skills, but also can promote communication between employees, increase communication between staff and teachers, managers.

(3) The system uses B / S structure, most of the work on the server side to achieve as much as possible to reduce the load on the client, making use of online training system more convenient. Developed using

(4) The three-tier system of MVC design model based on functional modules can be developed, so that the system has good scalability depending on the actual needs.

The online training system for electricity enterprises is set up on the Internet applications, database systems deployed in the information center server power plant, after a series of configuration on the server release. Employees can use their own computers or computer room at the plant, through the network login system, to participate in learning and training and assessment, not limited by time and place. 


\section{System Functions}

(1) Employees module:

Plant employees can choose courses and training programs in this module, after logging system, employees can be viewed online in accordance with the selection of courses and training programs to learn. When confronted with the problem, they can ask questions, answers will be conducted by a professional teacher. System also provides functionality to practice in order to enhance the effect of learning. In this module, employees can also view the news bulletin published by companies, get the latest training information.

(2) Learning arrangements

In this module, the teacher arranges training programs reasonable according to the actual situation of the various departments of the plant staff. Employee selection programs and courses to be checked, you can view employee learning plans and progress through the system.

(3) Course Management

This module is one of the most important online training module power plant systems, staff training materials obtained by the unified management of the module. Training materials may contain courseware, text, video, and other formats. Teacher training resources can be based on the latest acquisition of the plant, add, delete, course materials, so that employees always have access to the latest technical level of knowledge.

(4) Test Management

After learning courses, staffs have to exam at the front end of the training. Compliance is checked by inspection staff training and examinations. In this module, teacher training as needed to add content and modify exam, exam schedule according to the training program, the module under Questions, questions, and difficulty of constraints to achieve the automatic test paper function of the system, for papers and employee performance management.

(5) Management Information

This module is mainly used to announce the latest training information power plant. Training programs and plant constantly updated content; this module can publish the latest information. The bulletin information exist information Center database, easily lost, employees can view these announcements.

(6) User Management

The main function of this module is to manage employee information, online training in the use of the system when the power plant, employees must be logged in, you must have a user table that employee information system, and employees can successfully log in. In this module, the realization of employee information to add, delete, modify, and other operations.

(7) Q \& A module

Online learning when employees may encounter various problems professional can help to train teachers to consult this online form of questions, after the teacher receives training problems, answer questions, the answer is returned to the employee, promote exchanges between employees and teachers, quickly solve problems encountered in the training process, improve staff skills.

(8) System Management Module

System management module is mainly responsible for the plant to manage online training system, you need to have administrator privileges. The main features are the result of the analysis of training, the system user rights management, information management

\section{System Requirements}

(1) Server device

Server device uses 2.8GHZ, 8G memory, 1000M dual card configuration, the processor, memory, network basic online training system to meet the needs of the plant.

(2) Operating platform 
The underlying platform for all such should be able to provide good support for that piece in the center of the plant information system based on the use of Window XP plant application needs and hardware compatibility and other aspects of the requirements, the system comes with Microsoft IIS as the Web server.

(3) Database system

The system uses Microsoft SQL Server 2008 database, SQL Server 2008 has a powerful data management capabilities, and has good usability. It uses a layered communications system, the network and protocol layers separated, to ensure that the robustness of the application in different network environments.

\section{Summary}

With the rapid development of computer and Internet technology, network technology has gradually infiltrated all levels of social work and life. Traditional training methods because of its power plants consuming, laborious, inefficient and other defects have been unable to meet the power requirements of enterprise information, so the need for traditional training methods to adjust. Through access to information, combined with the actual situation of the work of the power plant, power plant online training system needs analysis. By the method of software engineering, design the overall functionality of the system. For each functional module of the system carried out a detailed analysis and process design. After conducting needs analysis, based on the functional requirements of the various subsystems of the database system design.

\section{Reference}

[1] Joyce M.Schmeeckle. Online Training: An Evaluation of the Effectiveness and Efficiency of Training Law Enforcement Personnel over the Internet[J]. Journal of Science Education and Technology.2003,(3):58-60.

[2] Abell Tamar. A New Look at Online Staff Training[J].Provider(Washington,D.C),2012,(3): 51-52,54.

[3] Chengyang Chang;Yang Fang;Qinglong Zhan. Design Principles and Methods of Activities for Teacher’s Online Training[J]. Networking and Digital Society,2009,1:258-260.

[4] Hsiao-Ya Chiu. Designing a Satisfaction-Oriented Option Analysis Framework to Support Organization Decision on Online Training Project[J]. Evolutionary Computation. 2008 : 3998-4005. 